\title{
Simulação matemática do comportamento teórico-hidráulico de microaspersores e microtubos ao longo da linha lateral
}

\section{Mathematical simulation of the theoretical-hydraulic behavior of microsprinklers and microtubes along of the lateral line}

\author{
Antonio Gebson PINHEIRO ${ }^{1}$; Jhon Lennon Bezerra da SILVA ${ }^{1}$; Douglas Alberto de Oliveira \\ SILVA $^{1}$; Pedro Henrique Dias BATISTA ${ }^{2}$; Joez André de Moraes RODRIGUES ${ }^{1}$; Marcos Vinícius \\ da SILVA ${ }^{2}$
}

\begin{abstract}
${ }^{1}$ Universidade Federal Rural de Pernambuco, Departamento de Engenharia Agrícola. E-mail: gebson10@ hotmail.com; jhonlennoigt@hotmail.com; douglasalbertosilva@hotmail.com; joezandre@hotmail.com

${ }^{2}$ Universidade Federal Rural de Pernambuco, Departamento de Ambiência. E-mail: giga_pedro@hotmail.com; morcolino_114@hotmail.com

*Autor correspondente: douglasalbertosilva@ hotmail.com
\end{abstract}

Resumo - A irrigação localizada caracteriza-se como o método de irrigação com alta eficiência na aplicação de água e insumos por meio da fertirrigação. Objetivou-se com o presente estudo avaliar o comportamento teórico-hidráulico de microaspersores e microtubos por meio de simulações matemáticas ao longo da linha lateral em sistema de irrigação, sendo o emissor em estudo da carborundum, cuja característica de bocal verde e claro. O trabalho foi desenvolvido através de simulações matemáticas através do Software Microsoft Excel ${ }^{\circledR}$ para um emissor tipo microaspersor e microtubos. Os valores estatísticos foram a média, desvio padrão, coeficiente de variação $(\mathrm{CV})$ e coeficiente de uniformidade estatísticos (CUE). A simulação com microaspersores e microtubos obteveram valores do CUE para a pressão e vazão variando entre 94,42 e 98,09\%, classificados como excelentes, apresentando-se de baixa variabilidade (CVs entre 1,00 e 5,00\%). Neste sistema, foi verificado que a uniformidade de aplicação de água ao longo da linha lateral está intimamente relacionada com a variação de pressão e vazão dos emissores. Os modelos matemáticos foram eficazes no dimensionamento do gradiente de pressão ao longo da linha lateral tanto para os microaspersores quanto para os microtubos.

Palavras-chave: Irrigação localizada; Modelos matemáticos; Baixo custo.

Abstract - Localized irrigation is characterized as the irrigation method with high efficiency in the application of water and inputs through fertigation. The objective of this study was to evaluate the theoretical-hydraulic behavior of microsprinklers and microtubes by means of mathematical simulations along the lateral line in an irrigation system, being the emitter in study of the carborundum, whose characteristic of green and clear nozzle. The work was developed through mathematical simulations through Excel $^{\circledR}$ Software for a microsprinklers type emitter and microtubes. The statistical values were the mean, standard deviation, coefficient of variation $(\mathrm{CV})$ and statistical uniformity coefficient (SUC). The simulation with microsprinklers and microtubes obtained SUC values for pressure and flow ranging from 94.42 to $98.09 \%$, classified as excellent, presenting low variability (CVs between 1,00 and 5,00\%). In this system, it was verified that the uniformity of water application along the lateral line is closely related to the variation of pressure 


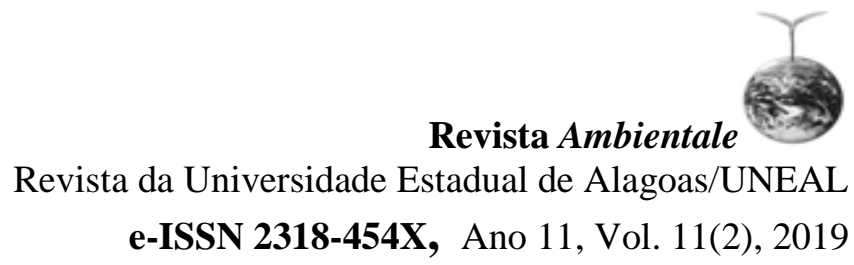

and flow of the emitters. The mathematical models were effective in sizing the pressure gradient along the lateral line for both microsprinklers and microtubes.

Keywords: Localized irrigation; Mathematical models; Low cost.

\section{Introdução}

O manejo adequado da irrigação é altamente significativo para uma produção agrícola em larga escala. Os sistemas de irrigação dentro da agricultura irrigada necessitam de um dimensionado hidráulico adequado e eficaz, em complementação, avalições hidráulicas devem ser constantes no intuito de atingir o uso eficiente de água e energia, por conseguinte ter-se um bom rendimento agrícola (NASCIMENTO et al., 2013; ANA, 2017; FRIZZONE, 2017; TESTEZLAF, 2017).

O sistema de irrigação localizada, por exemplo, é um dos que mais cresceu nas últimas décadas, e vem sendo largamente usado devido a vantagens como a maior economia e eficiência no uso da água e de insumos. É potencialmente o sistema de irrigação mais eficiente, com eficiência de aplicação de aproximadamente $90 \%$. A aplicação da água de irrigação neste sistema é realizada por emissores, que atuam sob pressão e possuem a vantagem de localizar o volume de água necessário as culturas, sendo próximo ao sistema radicular e em pequenas vazões, com baixa pressão e alta frequência (DANOLE \& PATIL, 2015; FRIZZONE, 2017).

$\mathrm{Na}$ irrigação localizada, o método por microaspersão, tem o objetivo aumentar a área molhada por um emissor, ao passo que os emissores são os mais sensíveis do sistema, podendo obstruir facilmente e comprometer a eficiência do sistema de irrigação. Todavia, o método visa assegurar o suprimento de água ao solo em período e quantidade adequada, e com uniformidade aceitável em toda unidade irrigada. A microaspersão é caracterizada hidraulicamente por sua pressão de serviço, variação e vazão nominal (SOUZA, 2005; FRIZZONE et al., 2012; SILVA et al., 2012; NASCIMENTO et al., 2016).

Visando o baixo custo e alto nível tecnológico do sistema de irrigação localizada, especificamente, destacam-se também o método de irrigação por microtubos como uma alternativa viável e prática. O microtubo é um tipo de gotejador feito de polietileno, com diâmetros entre 0,6 e 1,5 mm. Devido a alta sensibilidade à variação de temperatura e pressão, e susceptibilidade ao entupimento, emissores menos exigentes em mão-de-obra na instalação, resistentes a danos mecânicos e menos sensíveis às variações de pressão e temperatura se tornaram predominantes no mercado (SOUZA et al., 2005; ALVES et al., 2012).

Nesse contexto, objetivou-se avaliar e monitorar o comportamento teórico-hidráulico de um emissor tipo microaspersor e microtubos ao longo da linha lateral, através de simulação e modelagem matemática computacional para o controle e otimização de sistemas de irrigação localizada.

\section{Material e Métodos}

O trabalho foi realizado através de um dimensionamento teórico-hidráulico por meio de simulação matemática computacional para um sistema de irrigação localizada com um emissor tipo 
microaspersor e microtubos, pelo qual, foi avaliado o comportamento hidráulico do gradiente de pressão e do comprimento ao longo da linha lateral. A Tabela 1 destaca as características técnicas do emissor selecionado conforme o catálogo do fabricante, bocal verde claro de 1,0 $\mathrm{mm}$ e vazão (q) média de $51,12 \mathrm{~L} \mathrm{~h}^{-1}$ para uma pressão de serviço $(\mathrm{H})$ média de 20 mca.

Tabela 1. Características técnicas do emissor tipo microaspersor.

\begin{tabular}{cc}
\hline $\mathbf{H}(\mathbf{m c a})$ & $\mathbf{q}\left(\mathbf{L} \mathbf{~ h}^{-\mathbf{1}}\right)$ \\
\hline 10 & 38,99 \\
15 & 48,41 \\
20 & 52,01 \\
25 & 56,01 \\
30 & 60,19 \\
\hline
\end{tabular}

A Tabela 2 apresenta os dados de entrada para o desenvolvimento do projeto em relação ao microaspersor de bocal verde claro da carborundum, sendo realizadas as simulações hidráulicas para o dimensionamento da linha lateral, que por sua vez, utilizou-se de dados conforme o catálogo do fabricante.

Tabela 2. Dados de entrada para dimensionamento do emissor tipo microaspersor.

\begin{tabular}{lc}
\hline \multicolumn{2}{c}{ Dados de entrada - emissor microaspersor } \\
\hline Pressão de serviço (Ps) & $200 \mathrm{kPa}$ \\
$\mathrm{N}^{\circ}$ de microaspersores (N) & 30 \\
Espaçamento (S) & $3,3 \mathrm{~m}$ \\
Temperatura (T) & $20{ }^{\circ} \mathrm{C}$ \\
$\mathrm{hf}^{1}$ & $20 \%$ \\
$\mathrm{~m}$ & 1,75 \\
\hline \multicolumn{2}{c}{$\mathrm{hf}^{1}=$ Perda de carga na tubulação de múltiplas saídas; } \\
$\mathrm{m}=$ expoente da vazão na equação de perda de carga.
\end{tabular}

Para poder simular o perfil de pressão ao longo da linha lateral, adotou-se o modelo de dimensionamento proposto por $\mathrm{Wu} \&$ Gitlin (1975). De acordo com o modelo, os demais dados necessários para o dimensionamento do projeto foram calculados através de uma planilha do Software Microsoft Excel ${ }^{\circledR}$, como a descarga do microaspersor (q), com isso, pode-se encontrar a descarga total da linha lateral (Q), em $\mathrm{m}^{3} \mathrm{~s}^{-1}$, composta por 30 microaspersores, calculou-se sequencialmente a viscosidade cinemática da água $(\mathrm{V})$, em $\mathrm{m}^{2} \mathrm{~s}^{-1}$, para uma temperatura de $20{ }^{\circ} \mathrm{C}$, seguido da perda de carga $\left(\mathrm{hf}^{\mathrm{l}}\right)$, em mca, e o cálculo do fator de correção (Fc), dessa forma, através da divisão dos mesmos ( $\left.{ }^{1}{ }^{1} / \mathrm{Fc}\right)$ pode-se encontrar também a perda de carga no trecho (hf), em mca, e por fim, a perda de carga unitária $(\mathrm{J}), \mathrm{em} \mathrm{m} / \mathrm{m}$.

De posse dos dados calculados, logo obteve-se o diâmetro da linha lateral (D), dado em mm, bem como, o comprimento da linha lateral (L), em metros, e sua declividade, nesse caso, assumida como um valor igual a zero. 
Keller \& Karmeli (1974), em um dimensionamento de emissores, destacaram que a vazão depende diretamente da pressão de operação e pode ser determinada utilizando a Equação 1, onde k e x são parâmetros de ajuste. Quanto menor for o valor do expoente (x), a vazão será menos influenciada pelas variações de pressão. Assim, um emissor perfeito teria o expoente $\mathrm{x}=0$ (autocompensante).

$\mathrm{q}=\mathrm{k} \cdot \mathrm{H}^{\mathrm{x}}$

Em que,

$\mathrm{q}=$ Vazão do emissor $\left(\mathrm{L} \mathrm{h}^{-1}\right)$;

$\mathrm{k}=$ Constante de proporcionalidade que caracteriza cada emissor (adimensional);

$\mathrm{H}=$ Pressão de serviço do emissor $(\mathrm{m})$;

$\mathrm{x}=$ Expoente, determinado pelo regime de escoamento do emissor (adimensional).

A pressão varia em cada emissor ao longo de toda a linha lateral, considerando nesse sentido, por consequência a vazão também irá variar em cada emissor ao longo da linha, tornandose dependente do comportamento da pressão.

$\mathrm{Na}$ Equação 2, o fator de correção ( $\mathrm{Fc}$ ) proposto por Wu \& Gitlin (1975), usado nessa simulação ajudou a obter a perda de carga na tubulação de múltiplas saídas. Sendo que, as equações utilizadas anteriormente calcularam apenas a perda de carga numa tubulação.

$$
\mathrm{Fc}=\frac{1}{(\mathrm{~m}+1)}
$$

Em que,

$\mathrm{Fc}=$ Fator de correção (adimensional);

$\mathrm{m}=$ expoente da vazão na equação de perda de carga (adimensional).

Assim, foi possível estimar a variação de energia em cada microaspersor, de acordo com a Equação 3, proposta por Wu \& Gitlin (1975).

$\mathrm{H}=\mathrm{H}_{0}-\left(1-\left(1-\frac{1}{\mathrm{~L}}\right)^{\mathrm{m}+1}\right) \cdot \mathrm{hf}^{\mathrm{l}}$

Em que,

$\mathrm{H}=$ Pressão no início da linha lateral (mca);

1 = Posição do microaspersor;

$\mathrm{L}=$ Comprimento da linha lateral $(\mathrm{m})$;

$\mathrm{hf}^{1}=$ Perda de carga numa tubulação de múltiplas saídas (mca). 
O dimensionamento dos microtubos, correlaciona a vazão, comprimento e a pressão na entrada dos microtubos, com isso propõe-se o modelo da Equação 4 (SOUZA, 2005).

$$
\mathrm{H}=\frac{64 \cdot \mathrm{v} \cdot 4}{\pi \cdot 2 \cdot \mathrm{g}}\left(\frac{\mathrm{L} \cdot \mathrm{Q}}{\mathrm{d}^{4}}\right)+\frac{16}{\pi^{2} \cdot 2 \cdot \mathrm{g}}\left(\frac{\mathrm{Q}^{2}}{\mathrm{~d}^{4}}\right)+\frac{16}{\pi^{2} \cdot 2 \cdot \mathrm{g}}\left(\frac{\mathrm{K} \cdot \mathrm{Q}^{2}}{\mathrm{~d}^{4}}\right)
$$

Em que,

$\mathrm{H}=$ Carga piezométrica na entrada do microtubo $(\mathrm{kPa})$;

$\mathrm{Q}=$ Vazão do microtubo $\left(\mathrm{m}^{3} \mathrm{~s}^{-1}\right)$;

$\mathrm{g}=$ Aceleração da gravidade $\left(\mathrm{m} \mathrm{s}^{-2}\right)$;

$\mathrm{K}$ = Coeficiente de perda de carga localizada (adimensional);

$\mathrm{d}=$ Diâmetro interno do microtubo (m).

O modelo de Wu \& Gitlin (1975) é quem dá sequência a este dimensionamento, sendo que, para determinar o dimensionamento de microtubos foram fornecidos os dados de entrada conforme o fabricante, destacados na Tabela 3 .

Tabela 3. Dados de entrada utilizados para o dimensionamento de microtubo.

\section{Dados de entrada - microtubo}

\begin{tabular}{lc}
\hline Diâmetro da linha $\left(\mathrm{L}_{\mathrm{L}}\right)$ & $13,6 \mathrm{~mm}$ \\
$\mathrm{~N}^{\circ}$ de microtubos $\left(\mathrm{N}^{\circ}\right)$ & 30 \\
Espaçamento $(\mathrm{S})$ & $3,3 \mathrm{~m}$ \\
Temperatura $(\mathrm{T})$ & $20{ }^{\circ} \mathrm{C}$ \\
Vazão do microtubo (q) & $4 \mathrm{~L} \mathrm{~h}^{-1}$ \\
Gravidade $(\mathrm{g})$ & $10 \mathrm{~m} \mathrm{~s}^{-1}$ \\
$\mathrm{~m}$ & 1,75 \\
\hline
\end{tabular}

Os cálculos e modelos estatísticos para o dimensionamento dos microtubos também foram organizados e obtidos através do Software Microsoft Excel ${ }^{\circledR}$ por meio de planilhas eletrônicas. Os principais valores estatísticos apresentados foram da estatística descritiva, como a média, desvio padrão, coeficiente de variação $(\mathrm{CV})$ e, também o coeficiente de uniformidade estatístico (CUE).

O CV (\%) foi classificado quanto a sua variabilidade, conforme o critério de classificação de Warrick \& Nielsen (1980), sendo de baixa variabilidade valores de $<12 \%$, média variabilidade entre $12<\mathrm{CV}<60 \%$ e alta variabilidade valores de $\mathrm{CV}>60 \%$.

Através de regressão linear, verificou também a existência de uma relação funcional entre a "Vazão x Pressão", e através do coeficiente de determinação $\left(\mathrm{R}^{2}\right)$ avaliou-se a precisão. 


\section{Resultados e Discussão}

As simulações teórico-hidráulicas dos modelos matemáticos de Wu \& Gitlin (1975) e Souza (2005) destacaram um bom dimensionamento do gradiente de pressão ao longo da linha lateral para o emissor tipo microaspersor e microtubos no sistema de irrigação localizada, permitindo realizar análises estatísticas e comparativas dos resultados que pode servir, na prática, como base auxiliadora para a aplicação no campo de forma eficiente.

A Figura 1 apresenta a curva e equação característica da classificação do emissor tipo microaspersor conforme seu expoente e a variação de vazão em função da variação de pressão.

Figura 1. Curva e equação característica vazão-pressão do microaspersor.

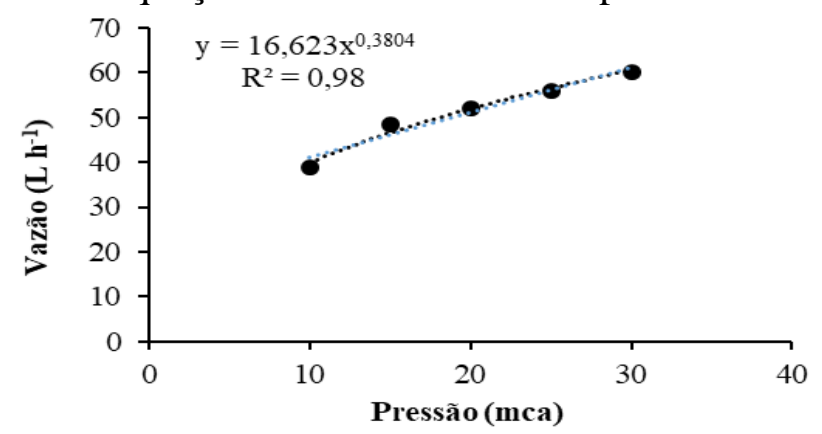

O modelo matemático mostrou-se altamente coerente, destacando uma equação de ajuste excelente, enfatizando uma relação funcional ótima entre a vazão e a pressão, com o mínimo de erro, apresentando $\mathrm{R}^{2}$ de 0,98 (Figura 1).

O expoente " $x$ " da equação foi de 0,3804, tal qual Frizzone et al. (2012) destacam que o emissor perfeito deve apresentar expoente " $x$ " igual a zero, o que anula o efeito da variação de pressão na vazão do emissor. $O$ expoente " $x$ " do emissor tipo microaspersor tende a zero, caracterizando-se como autocompensante por destacar uma menor sensibilidade do emissor à variação de pressão (SCHIMIDT, 2014).

$\mathrm{Na}$ Tabela 4 destacam-se os resultados da avaliação hidráulica por simulação matemática para 30 microspersores da marca carborundum de bocal verde claro. A linha lateral teve uma extensão de $99 \mathrm{~m}\left(\mathrm{~L}_{\mathrm{i}}\right)$, com o gradiente de pressão $\left(\mathrm{H}_{\mathrm{i}}\right)$ variando entre 22,57 e 18,93 mca, tendendo sempre a decrescer, sendo que a vazão $\left(\mathrm{q}_{\mathrm{i}}\right)$ variou entre 54,40 e $50,88 \mathrm{~L} \mathrm{~h}^{-1}$, destacando o mesmo comportamento. Desta forma, a vazão do sistema obviamente depende da pressão disponibilizada pela estação de bombeamento, logicamente se a pressão do sistema for afetada, consequentemente, também afetará a vazão (MARQUES \& CAMPECHE, 2012). 
Tabela 4. Avaliação teórico-hidráulica por simulação matemática da pressão-vazão dos 30 microaspersores.

\begin{tabular}{|c|c|c|c|}
\hline \multicolumn{4}{|c|}{ Simulação microaspersor: 1 - 30} \\
\hline $\mathbf{N}^{\circ}$ & $\mathbf{L}_{\mathbf{i}}(\mathbf{m})$ & $\mathrm{H}_{\mathrm{i}}(\mathbf{m c a})$ & $q_{i}\left(L_{h} h^{-1}\right)$ \\
\hline 1 & 3,3 & 22,57 & 54,40 \\
\hline 2 & 6,6 & 22,24 & 54,09 \\
\hline 3 & 9,9 & 21,92 & 53,80 \\
\hline 4 & 13,2 & 21,63 & 53,52 \\
\hline 5 & 16,5 & 21,35 & 53,26 \\
\hline 6 & 19,8 & 21,09 & 53,02 \\
\hline 7 & 23,1 & 20,86 & 52,79 \\
\hline 8 & 26,4 & 20,64 & 52,58 \\
\hline 9 & 29,7 & 20,43 & 52,38 \\
\hline 10 & 33 & 20,24 & 52,19 \\
\hline 11 & 36,3 & 20,07 & 52,02 \\
\hline 12 & 39,6 & 19,91 & 51,87 \\
\hline 13 & 42,9 & 19,77 & 51,73 \\
\hline 14 & 46,2 & 19,64 & 51,60 \\
\hline 15 & 49,5 & 19,53 & 51,48 \\
\hline 16 & 52,8 & 19,43 & 51,38 \\
\hline 17 & 56,1 & 19,33 & 51,29 \\
\hline 18 & 59,4 & 19,26 & 51,21 \\
\hline 19 & 62,7 & 19,19 & 51,14 \\
\hline 20 & 66 & 19,13 & 51,08 \\
\hline 21 & 69,3 & 19,08 & 51,03 \\
\hline 22 & 72,6 & 19,04 & 50,99 \\
\hline 23 & 75,9 & 19,01 & 50,96 \\
\hline 24 & 79,2 & 18,98 & 50,93 \\
\hline 25 & 82,5 & 18,96 & 50,91 \\
\hline 26 & 85,8 & 18,95 & 50,90 \\
\hline 27 & 89,1 & 18,94 & 50,89 \\
\hline 28 & 92,4 & 18,94 & 50,89 \\
\hline 29 & 95,7 & 18,93 & 50,88 \\
\hline 30 & 99 & 18,93 & 50,88 \\
\hline & & 19,93 & 51,87 \\
\hline Des & adrão & 1,11 & 1,08 \\
\hline & & 5,00 & 2,00 \\
\hline & (\%) & 94,42 & 97,90 \\
\hline
\end{tabular}


O gradiente de pressão e vazão apresentaram comportamento de baixa variabilidade, conforme destacaram os respectivos valores do CV, de 5,00 e 2,00\% (WARRICK \& NIELSEN, 1980). O CUE para a pressão e vazão foram de 94,42 e 97,90\%, respectivamente (Tabela 4). Os valores do CUE mostraram-se "excelentes", para CUE $\geq 90 \%$ conforme a classificação de Bralts (1986).

A Figura 2 apresenta a relação da vazão-pressão em função dos emissores distribuídos na linha lateral, conforme a simulação matemática obtida anteriormente. Assim, pode-se observar o comportamento hidráulico da vazão e da pressão em função dos emissores, sendo que, a relação vazão-pressão decresceu ao longo da linha lateral.

Figura 2. Relação vazão-pressão na linha lateral em função da posição dos microaspersores.

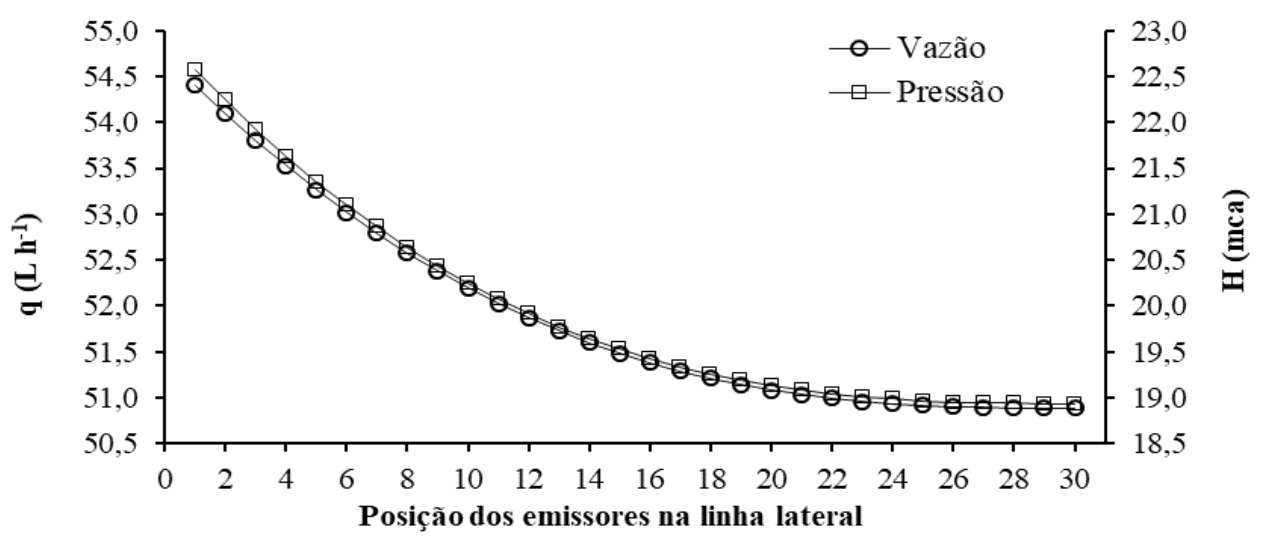

A quantidade de água aplicada e a uniformidade de distribuição pelos emissores são informações importantes para o dimensionamento e manejo racional de um sistema de irrigação localizada. Neste sistema a uniformidade de aplicação de água ao longo da linha lateral está intimamente relacionada com a variação de pressão e vazão dos emissores, a qual é uma consequência das perdas de energia por atrito e pelas inserções dos emissores, do ganho ou perda de energia devido à topografia da superfície do solo e da qualidade da matéria prima, e também dos processos de fabricação dos emissores (ALMEIDA, 2008).

A Tabela 5 destaca a avaliação hidráulica por simulação matemática para 30 microtubos de acordo com Souza (2005), sendo que o gradiente de pressão variou de 4,60 a 4,33\% e o comprimento do microtubo entre 1,08 e $1,00 \mathrm{~m}$. 
Tabela 5. Avaliação teórico-hidráulica por simulação matemática em função do comprimento e gradiente de pressão de 30 microtubos.

\begin{tabular}{|c|c|c|}
\hline \multicolumn{3}{|c|}{ Simulação dos microtubos: 1 - 30} \\
\hline Ordem (l) & $\mathbf{H}_{\mathrm{i}}$ (mca) & $\mathbf{L}_{\mathbf{i}}(\mathbf{m})$ \\
\hline 1 & 4,60 & 1,08 \\
\hline 2 & 4,58 & 1,07 \\
\hline 3 & 4,55 & 1,06 \\
\hline 4 & 4,53 & 1,06 \\
\hline 5 & 4,51 & 1,05 \\
\hline 6 & 4,49 & 1,04 \\
\hline 7 & 4,47 & 1,04 \\
\hline 8 & 4,46 & 1,03 \\
\hline 9 & 4,44 & 1,03 \\
\hline 10 & 4,43 & 1,03 \\
\hline 11 & 4,41 & 1,02 \\
\hline 12 & 4,40 & 1,02 \\
\hline 13 & 4,39 & 1,02 \\
\hline 14 & 4,38 & 1,01 \\
\hline 15 & 4,37 & 1,01 \\
\hline 16 & 4,37 & 1,01 \\
\hline 17 & 4,36 & 1,01 \\
\hline 18 & 4,35 & 1,01 \\
\hline 19 & 4,35 & 1,00 \\
\hline 20 & 4,34 & 1,00 \\
\hline 21 & 4,34 & 1,00 \\
\hline 22 & 4,34 & 1,00 \\
\hline 23 & 4,33 & 1,00 \\
\hline 24 & 4,33 & 1,00 \\
\hline 25 & 4,33 & 1,00 \\
\hline 26 & 4,33 & 1,00 \\
\hline 27 & 4,33 & 1,00 \\
\hline 28 & 4,33 & 1,00 \\
\hline 29 & 4,33 & 1,00 \\
\hline 30 & 4,33 & 1,00 \\
\hline Média & 4,40 & 1,02 \\
\hline Desvio Padrão & 0,08 & 0,02 \\
\hline CV $(\%)$ & 1,00 & 2,00 \\
\hline CUE $(\%)$ & 98,09 & 97,70 \\
\hline
\end{tabular}


O gradiente de pressão e vazão apresentaram também comportamento de baixa variabilidade, confirmado pelos valores do CV, entre 1,00 e 2,00\% (WARRICK \& NIELSEN, 1980). O CUE apresentou para a pressão e vazão dos microtubos valores entre 98,09 e 97,70\%, respectivamente, que conforme os critérios de classificação de Bralts (1986), foram excelentes (Tabela 4).

A Figura 3 apresenta a relação do gradiente de pressão e do comprimento dos microtubos em função da sua posição. Com isso, foi possível avaliar a diminuição da pressão ao longo da linha lateral, por sua vez, o comprimento obteve o mesmo comportamento hidráulico, necessariamente esperado, assim, a vazão se manterá o mais constante possível ao longo da linha.

Figura 3. Correlação do gradiente de energia e comprimento dos microtubos ao longo da linha lateral, sob regime laminar.

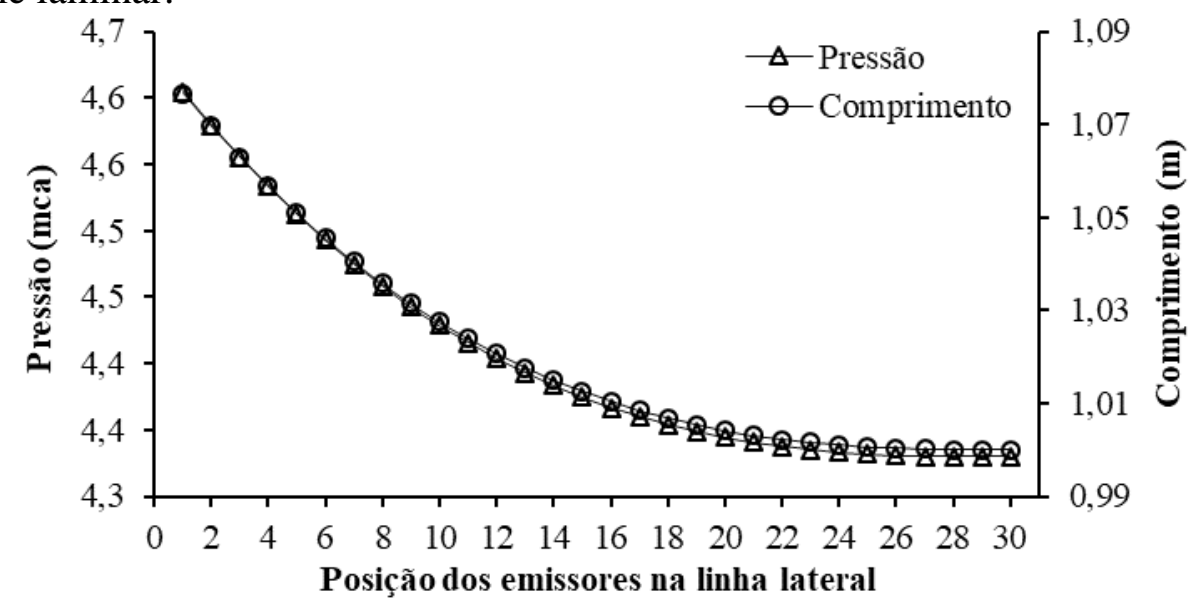

Souza et al. (2005) enfatizaram que o comprimento variado dos microtubos gotejadores visa minimizar os efeitos da perda de carga ao longo da linha lateral e da perda de carga localizada devido a inserção do microtubo na linha, de forma a manter uniforme a vazão nos microtubos emissores. Além disso, quanto maior o valor do coeficiente de perda de carga $(\mathrm{K})$, menor a variação no comprimento dos microtubos ao longo da linha.

\section{Conclusões}

As aplicações teórico-hidráulicas dos modelos matemáticos foram eficazes no dimensionamento do gradiente de pressão ao longo da linha lateral tanto para os microaspersores quanto para os microtubos.

As simulações matemáticas podem ser essenciais para um dimensionamento hidráulico adequado e eficaz dos sistemas de irrigação localizada, destacando praticidade e baixo custo. 
Contudo, é necessário validar tais simulações matemáticas em campo para destacar a boa eficiência dos modelos e do emissor tipo microaspersor e microtubo.

\section{Conflito de interesse}

Os autores declararam que não existem interesses conflitantes.

\section{Agradecimentos}

À Universidade Federal Rural de Pernambuco e o Programa de Pós-Graduação em Engenharia Agrícola; O presente trabalho foi realizado com apoio da Coordenação de Aperfeiçoamento de Pessoal de Nível Superior - Brasil (CAPES) - Código de Financiamento 001.

\section{Referências}

ALMEIDA, C. D. G. C. Microaspersor com microtubos: um novo conceito hidráulico na irrigação localizada. 2008. Tese (Doutorado em Irrigação e Drenagem) - Escola Superior de Agricultura Luiz de Queiroz, Universidade de São Paulo, Piracicaba, 2008.

ALVES, D. G.; PINTO, M. F.; SALVADOR, C. A.; ALMEIDA, A. C. D. S.; ALMEIDA, C. D. G. C. D.; \& BOTREL, T. A. Modelagem para o dimensionamento de um sistema de microirrigação utilizando microtubos ramificados. Revista Brasileira de Engenharia Agrícola e Ambiental, v. 16, p. 125-132, 2013.

ANA. AGÊNCIA NACIONAL DE ÁGUAS (Brasil). Atlas irrigação: uso da água na agricultura irrigada / Agência Nacional de Águas. -- Brasília: ANA, 2017, 86 p.

BRALTS, V. F. Field performance and evaluation. In: NAKAYAMA, F. S.; BUCKS, D. A. (Ed.). Trickle of irrigation for crop production. Elsevier, chap. 3, p. 216-240, 1986.

DANOLE, A. S; PATIL, B. M. Evaluation of local head loss for non pressure compensation online emitters and pressure compensating inline emitters in drip irrigation laterals. International Journal of Emerging Research in Management \& Technology, v. 4, p. 9-16, 2015.

FRIZZONE, J. A. Os métodos de irrigação. Departamento de Engenharia de Biossistemas, ESALQ/USP, 2017. Disponível em:

<http://www.leb.esalq.usp.br/leb/disciplinas/Frizzone/LEB_1571/TEXTO_COMPLEMENTAR_1_ -_METODOS_DE_IRRIGACAO.pdf>. Acesso em: 03 de jun. 2019.

FRIZZONE, J. A; LOURENÇO, P. S.; REZENDE, R.; FARIA, M. A. Microirrigação, gotejamento e microaspersão. Maringá: Editora EDUEM, 2012. 365 p. 
KELLER, J.; KARMELI, D. Trickle irrigation design parameters. Transaction of the ASAE, v. 17, p. 678-68, 1974.

MARQUES, M. A. D.; \& CAMPECHE, L. F. D. S. M. Caracterização hidráulica de emissores e testes de uniformidade de distribuição do sistema de irrigação por mini aspersão. Revista

Semiárido De Visu, v. 2, p. 162-175, 2012.

NASCIMENTO, A. K. S.; MELO SOUZA, R. O. R.; LIMA, S. C. R. V.; CARVALHO, C. M.; ROCHA, B. M.; NASCIMENTO LEITE, K. Desempenho hidráulico e manejo da irrigação em sistema irrigado por microaspersão. Revista Brasileira de Agricultura Irrigada, v. 3, p. 39-45, 2013.

NASCIMENTO, R. C.; MEDEIROS, P. R. F.; SANTOS, G. S.; ALENCAR, E. C. P. V.; GONÇALVES, D. R.; MARTINS, A. P. F. Dinâmica da obstrução de emissores usados na cultura da uva utilizando água salina de poço subterraneo. Irriga, v. 21, p. 491-502, 2016.

SCHIMIDT, A. P. R. A. Efeito da espessura da parede no desempenho hidráulico de mangueiras gotejadoras. 2014. 47 f. Dissertação (Mestrado em Agronomia/Irrigação e Drenagem) - Faculdade de Ciências Agronômicas, Universidade Estadual Paulista "Júlio de Mesquita Filho", Botucatu - SP, 2014.

SILVA, L. P.; SILVA, M. M.; CORREA, M. M.; SOUZA, F. C. D.; SILVA, E. F. F. E. Desempenho de gotejadores autocompensantes com diferentes efluentes de esgoto doméstico.

Revista Brasileira de Engenharia Agrícola e Ambiental, v. 16, p. 480-486, 2012.

SOUZA, I. H.; DE ANDRADE, E. M.; \& DA SILVA, E. L. Avaliação hidráulica de um sistema de irrigação localizada de baixa pressão, projetado pelo software. Engenharia Agrícola, v. 25, p. 264$271,2005$.

SOUZA, J. D.; DENÍCULLI, W.; BATISTA, R. O.; VAL, J. C. C.; \& MATOS, A. D.

Caracterização hidráulica de microaspersor aplicando água limpa, água residuária de avicultura e de bovinocultura. Engenharia na Agricultura, v. 13, p. 161-172, 2005.

SOUZA, R. R. M. Modelagem, desenvolvimento de software para dimensionamento, e avaliação de sistemas de irrigação por gotejamento com microtubos. $2005.100 \mathrm{f}$. Tese de Doutorado. Piracicaba, ESALQ/USP. 2005.

TESTEZLAF, R. Irrigação: métodos, sistemas e aplicações. Campinas: FEAGRI, 2017, 215 p.

WARRICK, A. W., NIELSEN, D. R. Spatial variability of soil physical properties in the field. In: HILLEL, D., ed. Applications of soil physics. New York, Academic Press. 1980. 350 p.

WU, I. P.; GITLIN, H. M. Energy gradient line for drip irrigation laterals. Journal of the Irrigation and Drainage Division, v. 101, p. 323-326, 1975. 